\title{
Improvement of Compressive Deformation Theory of Carpets
}

\section{and Its Application to Carpet Woven with Compressible Yarns}

\author{
By Kazutomi Kimura* and Sueo Kawabata**, Members, TMSJ \\ * Sakai Branch of Textile Research Institute, Osaka Prefecture, Osaka. \\ **Department of Polymer Chemistry, Kyoto University, Kyoto.
}

Based on the Journal of the Textile Machinery Society of Japan, Transactions, Vol. 24, No. 11, T207-214 (1971)

\begin{abstract}
The compressive deformation theory of carpets presented in the previous paper is improved in this paper.

At first, the compressive deformation of the carpet yarns by a lateral pressure is introduced in this theory.

Secondarily, the bending property of the yarn is used for theoretical calculation instead of the property of the fiber.

By these two improvements, the theory can extend its validity and becomes applicable to calculating the compressive property of the tufted and axminster carpets woven from twofolded yarns.

The experimental curves of the relation between compression and deformation of those tufted and axminster carpets have been compared with theoretical ones with fairly good agreement.
\end{abstract}

\section{Introduction}

The previous article ${ }^{[1]}$ proved that the compressive deformation theory proposed by the authors was applicable with high accuracy to calculating compressive properties of flocked carpets comparatively simple in structure and of cut pile carpets having piles of single yarns. Considerable errors were found, however, in the theoretical prediction of (1) the hysteresis of compressive deformation curves and (2) the thickness of carpets when piles had been fallen down.

For the compressive deformation of cut pile carpets having piles of two-fold yarns the theory required further improvement.

The major cause of errors is due to the fact that the physical properties of carpets were calculated from those of single fibers without taking into consideration the properties of yarns (fiber assemblies) used for piles.

This article improves the compressive deformation model and the theory so as to enable the properties of yarns to be easily incorporated in it.
The result shows that if the measured bending rigidity and compression of pile yarns are used for theoretical calculation, the theory gives high accuracy and can be applied to various structures of carpets.

\section{Theory}

When a carpet is compressed, it will show such a compressive deformation curve as illustrated in Fig. 1. If the length of piles is $l$, the initial thickness of the carpet $\overline{A o}$ is $l \cos \beta$ where $\beta$ is the angle of inclination.

The compression process can be divided into the following three stages of deformation ${ }^{[2]}$ :

(1) Bending deformation region

A region in which only the bending deformation is effective (until neighboring piles come in contact).

(2) Mixed region of bending and compressive deformations

A region in which both the bending and the compressive deformations are effective, i.e., the concurrence of the bending deformation of piles which 


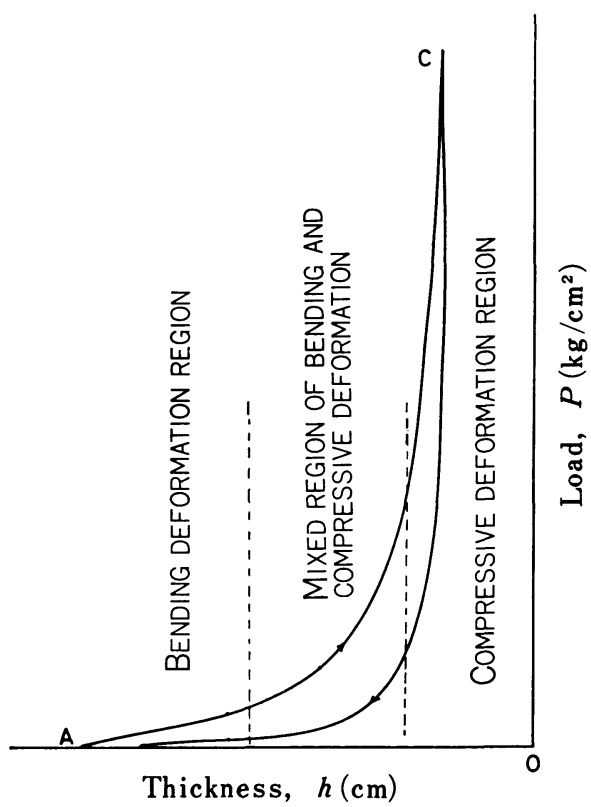

Fig. 1 Compressive deformation curves of carpet

do not come in contact with neighboring piles and the compressive deformation of piles which come in contact.

(3) Compressive deformation region

A region in which only the compressive deformation is effective (after all the piles are completely fallen down).

Only item (1) above mentioned was discussed in the previous article. Items (2) and (3) will be studied here for the improvement of the theory.

2-1. Calculation for Bending Deformation Region

The theory proposed in the previous article ${ }^{[1]}$ can be summed up as follows: Assuming that the cut pile length is $l(\mathrm{~cm})$, the compressive angle $\alpha\left(^{\circ}\right)$, the pile inclination angle $\beta\left(^{\circ}\right)$, the stitch $S$ (number of piles/in), the gauge G(in), the number of fibers having the diameter of $\mathrm{d}(\mathrm{cm}) n_{y}$, the bending rigidity of single fiber $\mathrm{EI}\left(\mathrm{g} . \mathrm{cm}^{2}\right)$, then the relationship between the pile density $n$ (number of piles $/ \mathrm{cm}^{2}$ ), the (a)
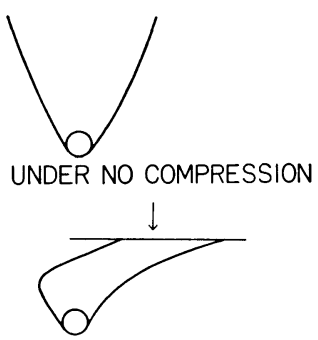

(b) UNDER COMPRESSION

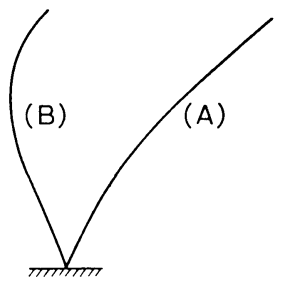

(c) PILE MODELED
Fig. Compressive configuration maximum compressive angle $\alpha_{m}\left({ }^{\circ}\right)$, the compressive load $P_{A}\left(\mathrm{~g} / \mathrm{cm}^{2}\right)$ in case of compressive configuration (A) shown in Fig. 2, and the pile thickness of carpet $h(\mathrm{~cm})$, is given by the following equations. For the sake of simplicity, however, the coefficient of friction between fibers of piles and the compressing plates is assumed to be 0.

$$
\begin{aligned}
& \alpha_{m}=\cos ^{-1}\left(\frac{2 n_{y} \times d^{2} \times S}{2.54^{2} \times G}\right) \\
& n=\frac{2 S}{2.54^{2} \times G} \\
& P_{A}=\frac{n_{y} n E I}{l^{2}}\left(\int_{\phi_{B}}^{\frac{\pi}{2}} \frac{d \phi}{\sqrt{1-K^{2} \sin ^{2} \phi}}\right)^{2} \\
& h=\sqrt{\frac{n_{y} n E I}{P_{A}}}\left(2 \int_{\phi_{B}}^{\frac{\pi}{2}} \sqrt{1-K^{2} \sin ^{2} \phi} d \phi(1)\right. \\
& \left.\quad \int_{\phi_{B}}^{\frac{\pi}{2}} \frac{d \phi}{\sqrt{1-K^{2} \sin ^{2} \phi}}\right) \\
& \text { where } \quad K=\sin \frac{\alpha}{2}, \phi_{B}=\sin ^{-1}\left\{\left(\frac{\sin \left(\frac{\beta}{2}\right)}{\sin \left(\frac{\alpha}{2}\right)}\right)\right\}
\end{aligned}
$$

In general, the cut pile carpets are likely to show both the compressive configurations (A) and (B) concurrently.

If the probabilities of compressive configurations $(\mathrm{A})$ and (B) are $N_{A}$ and $N_{B}$, respectively, the actual compressive load $P\left(\mathrm{~g} / \mathrm{cm}^{2}\right)$ will be

$$
\begin{aligned}
& P \simeq\left(N_{A}+8 N_{B}\right) P_{A} \\
& N_{A}+N_{B}=1
\end{aligned}
$$

Due to the construction of woven carpet, one half of piles will take the configuration (A) as shown in Fig. 2(C) and another half will take the configuration (B). Accordingly, both $N_{A}$ and $N_{B}$ can be assumed to be $1 / 2$.

Substitute eq. (3) into eq. (5), and

$$
\begin{aligned}
& P= \\
& \quad-\frac{n_{y} n E I}{l^{2}} \times\left(N_{A}+8 N_{\mathrm{B}}\right) \times\left(\int_{\phi_{B}}^{\frac{\pi}{2}} \frac{d \phi}{\sqrt{1-K^{2} \sin ^{2} \phi}}\right)^{2}
\end{aligned}
$$

Then, substitute eq. (3) into eq. (4), and

$$
h=l \times\left(\frac{2 \int_{\phi_{B}}^{\frac{\pi}{2}} \sqrt{1-K^{2} \sin ^{2} \phi} d \phi}{\int_{\phi_{B}}^{\pi^{-}} \frac{d \phi}{\sqrt{1-K^{2}} \sin ^{2} \phi}}-1\right)
$$


Hence the relation between $P$ and $h$ can be calculated by varying $\alpha$ from $\beta\left(^{\circ}\right)$ to $\alpha_{m}\left(^{\circ}\right)$.

Since the method to calculate the properties of the carpet from (1) the properties $(d, E I)$ of single fibers and (2) the number $\left(n_{y}\right)$ of fibers constituting the yarn is not applicable to bulky yarns, a new method is devised in which the properties of yarns (fiber assemblies) can be incorporated.

In order to incorporate actual properties of pile yarns, the relation between the compressive load of the pile yarns and the diameter of the piles is employed. Now, assuming the apparent diameter of pile yarns to be $D c$, the maximum compressive angle in the bending deformation region, is from eq. (1) in the previous article:

$$
\alpha_{m}=\cos ^{-1}\left(D_{c} \times \sqrt{n}\right)
$$

The compressive load $P$ is from eq. (7)

$$
P=\frac{n E I}{l^{2}} \times\left(N_{A}+8 N_{\mathrm{B}}\right) \times\left(\int_{\phi_{B}}^{-\frac{\pi}{2}} \frac{d \phi}{\sqrt{1-K^{2} \sin ^{2} \phi}}\right)^{2}
$$

Here, the loading curve and the unloading curve are calculated separately, by substituting $E I$ as the measured value of the bending rigidity of pile yarns into eq. (10) in case of the compressive process or substituting $E I$ in case of recovery process. The method to calculate $E I$ and $E I$ will be described later.

2-1-1. Measurement of bending rigidity of pile yarns

When a pile yarn is compressed with load $P$ as illustrated in Fig. 3(a), it will deform by $\left(D-D^{\prime}\right)$ and take the configuration (b). Supposing the pile yarn as an elastic rod having the pile length $l$ and the bending rigidity $E I$, the following equation will be led in the light of the so-called elastic theory:

$$
\frac{P}{2}=\frac{E I}{l^{2}}\left(\int_{0}^{\frac{\pi}{2}} \frac{d \phi}{\sqrt{1-K^{2} \sin ^{2} \phi}}\right)^{2}
$$

where $K=\sin \pi / 4$

The relation between $D$ and $l$ will be

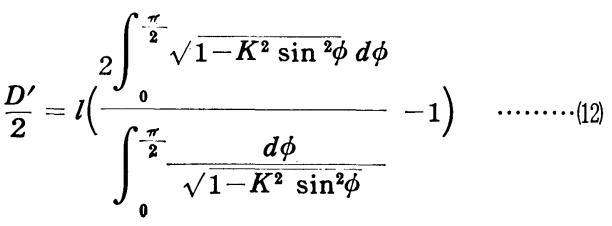

From eqs. (11) and (12), eq. (13) will be obtained:

$$
E I=0.174 \times D^{\prime 2} \times P
$$

Since the relation between $D^{\prime}$ and $P$ can be easily measured with a compressive tester, $E I$ as the bending rigidity under load and $E I$ as the bending rigidity under

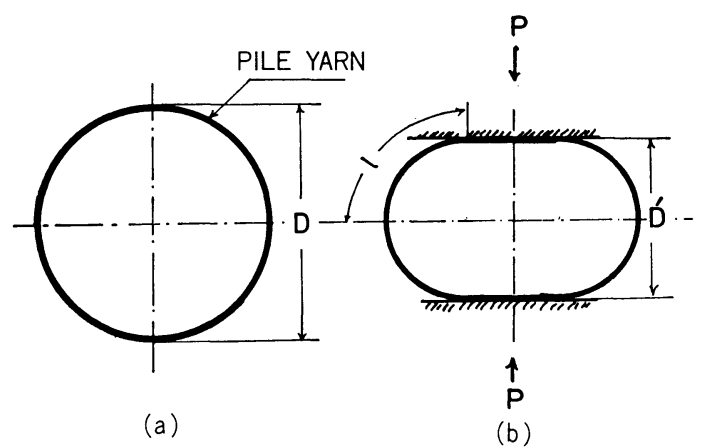

Fig. 3 How to measure the bending

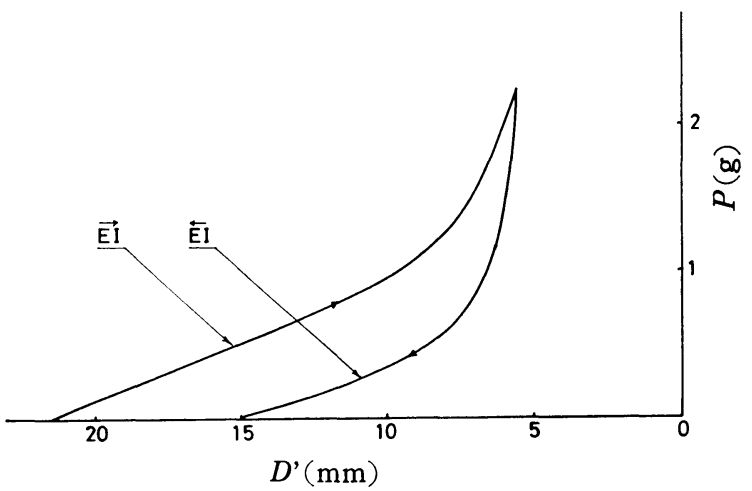

Fig. 4 Measured curves to calculate EI

unloading can be calculated from measured values and eq. (13) as illustrated in Fig. 4. However, EI which is accompanied by this hysteresis is not important any more from physical view point. It should be considered as an equivalent value converted into an elastic substance. When $P$ or $\left(D-D^{\prime}\right)$ is within a small range, eq. (11) will be invalid. So, when the condition illustrated in Fig. 3(b) is reached, $E I$ is measured.

2-2. Mixed Region of Bending and Compressive Deformation

The relation between load $P(\mathrm{~g} / \mathrm{cm})$ and thickness $h(\mathrm{~cm})$ in this mixed region will be improved.

The yarn diameter decreases with compressive load, and its cross section reaches such a rectangle as illustrated in Fig. 14 in the previous article.

Assuming that the yarn is full of fibers with highest density (Fig. 5), that the thickness of the yarn is $D c^{\prime}$ and the diameter of the pile yarn imposed with a compressive load is $D c p$, the following equation is obtained.

$$
\frac{\pi}{4}-D_{c p}^{2}=I_{n} D_{c}^{\prime}
$$

where $I_{n}=\frac{1}{\sqrt{n}}$

$$
D_{c}^{\prime}=\frac{\pi}{4} \times \sqrt{n} \times D_{c p}^{2}
$$



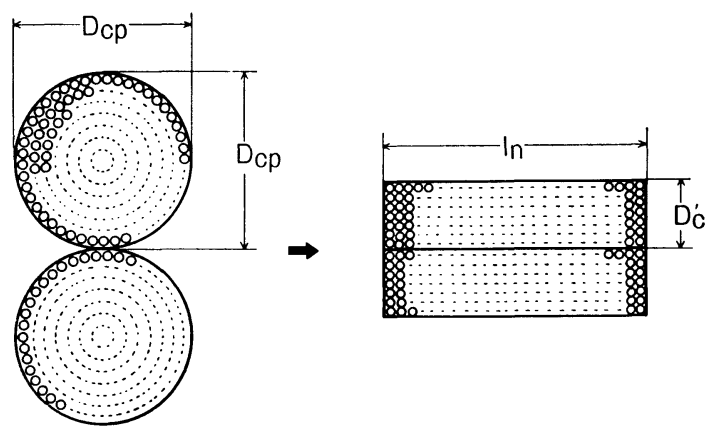

Fig. 5 Effect of collapse of pile yarns

When a yarn is put on a compressive tester, it will not collapse while keeping the circular cross section but will take the elliptical cross section. Assuming the long diameter of the ellipse is $2 \mathrm{a}(=\mathrm{In})$ and the short diameter is $2 \mathrm{~b}$, we have $2 \mathrm{~b}=D^{\prime \prime}{ }_{c}=4 / \pi D^{\prime}{ }_{c}=1.27 D^{\prime}{ }_{c}$ from $\mathrm{ab} \pi=\mathrm{In}$ $D^{\prime}{ }_{c}$. Namely, the thickness $\left(D^{\prime \prime}{ }_{c}\right)$ of the yarn adjusted is

$$
D_{c}^{\prime \prime}=1.27 \times D_{c}^{\prime}
$$

Accordingly, the maximum compressive angle $\alpha_{m}$ is

$\alpha_{m}=\cos ^{-1}\left(D^{\prime \prime}{ }_{c} \times \sqrt{n}\right)$

Then, from eqs. (15) and (16),

$$
\alpha_{m} \div \cos ^{-1}\left(D_{c p}{ }^{2} \times n\right)
$$

The relation between load $P\left(\mathrm{~g} / \mathrm{cm}^{2}\right)$ and thickness $h(\mathrm{~cm})$ can be derived from eqs. (8) and (10) in the same manner as the previous article,

$$
\begin{aligned}
& P=n E I\left(\int_{\phi_{B}}^{\frac{\pi}{2}} \frac{d \phi}{\sqrt{1-K^{2} \sin ^{2} \phi}}\right)^{2} \times\left(N_{A}+8 N_{\mathrm{B}}\right) \\
& \times \frac{1}{l_{2}{ }^{2}} \quad \quad \cdots \cdots \cdots(18) \\
& h=\left(\frac{2 \int_{\phi_{Q}}^{\frac{\pi}{2}} \sqrt{1-K^{2} \sin ^{2} \phi} d \phi}{\int_{\frac{\pi}{2}}}-1\right) l_{2}+\left(\cos \alpha_{m}\right) l_{1} \ldots(19) \\
& \int_{\phi_{B}}^{-\frac{\pi}{2}} \frac{d \phi}{\sqrt{1-K^{2} \sin ^{2} \phi}} \\
& l=l_{1}+l_{2} \\
& \text {.........(20) } \\
& \text { where } K=\sin \frac{\alpha_{m}}{2}, \quad \phi_{\beta}=\sin ^{-1}\left(\frac{\sin \frac{\beta}{2}}{\sin \frac{\alpha_{m}}{2}}\right)
\end{aligned}
$$

When $D_{c p}$ of eq. (17) is constant, the maximum compressive angle $\alpha_{m}$ is constant. So when $l_{1}$ is varied from 0 to $l$ the relation between $P$ and $h$ can be calculated.

However, when the carpet is compressed in the actual usage, $D_{c p}, D^{\prime \prime}{ }_{c}$ varies. So the calculating method is as follows:

2-2-1. Calculation of compressive load imposed on a yarn Assuming that the compressive load is $P\left(\mathrm{~g} / \mathrm{cm}^{2}\right)$, the

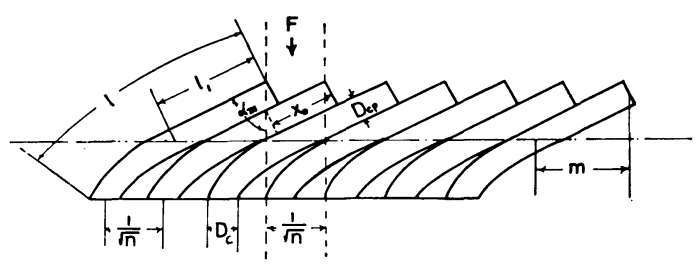

Fig. 6 Model of mixed region

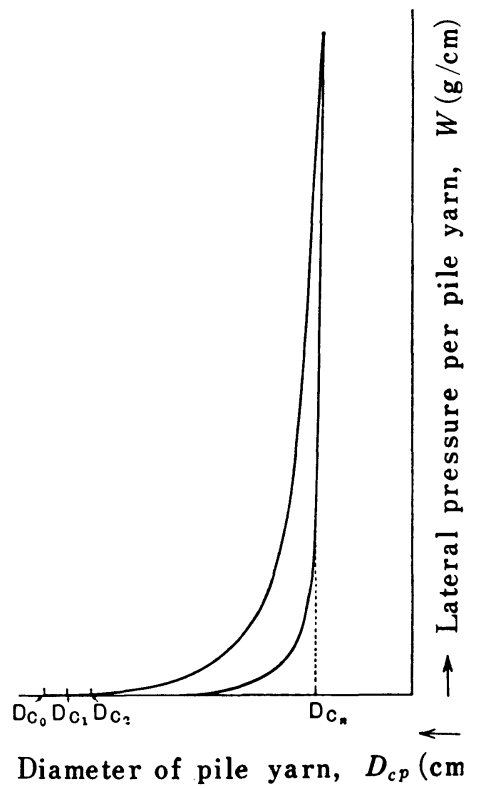

Fig. 7 Compressive property per pile yarn

pile density, $n$ (number of yarns $/ \mathrm{cm}^{2}$ ), the compressed area $S q\left(\mathrm{~cm}^{2}\right)$, and the diameter of compressing plates $2 \mathrm{R}$, then the compressive force per pile (Fig. 6) is

$$
F=\frac{\pi R^{2} P}{S_{q 0} \times n}
$$

Since

$$
F=\frac{P}{n}
$$

The length $X_{0}$, the straight part carrying the load (Fig. 6) is

$$
X_{0}=\frac{1}{\sqrt{n} \sin \alpha_{m}}
$$

Also, the projection of $l_{1}$ to the surface is

$$
m=l_{1} \sin \alpha_{m}
$$

The lateral pressure $W(\mathrm{~g} / \mathrm{cm})$ per unit length $(1 \mathrm{~cm})$ imposed on $X_{0}$ is

$$
W=\frac{F \sin \alpha_{m}}{X_{0}}
$$


From eqs. (21), (22), and (23),

$$
W=\frac{P \sin ^{2} \alpha_{m}}{\sqrt{n}}
$$

2-2-2. Method to calculate the diameter of the yarn under compression

To calculate the relation between $P$ and $h$ from eqs. (17) (18), (19) and (20), it is necessary to decide the values of $l_{1}$ and $D_{c p}$, which depend upon $P$ and $h$.

Accordingly, using eq. (26) in addition to eqs. (17) to (20), the relation is graphically decided as follows by measured compressive property of pile yarns. However, the range of $l_{1}$ is assumed to be $0 \leqq l_{1} \leqq l$ and the range of $D_{c p}, D_{c o} \geqq D_{c p} \geqq D_{c n}$ from Fig. 7 .

In other words, taking $l_{1}$ as a parameter and $D c p$ as a variable, $W$ is calculated as a function of $D_{c p}$ by eqs. (17), (18), (20) and (26). Then the relation between $l_{1}, D_{c p}$ and $W$ can be plotted as a function of $l_{1}$ as illustrated in Fig. 8 .

Since the relation between $D_{c p}$ and $W$ was plotted as a curve through the compression test of yarns (solid line in Fig. 8), the crossing point of the group of dotted lines with the solid lines can afford the relation between $D_{c p}$ and $W$. From $D_{c p}$ and $l_{1}$, the relation between $h$ and $P$ can be calculated. The same procedure is repeated for each crossing point, and when these points are connected, the compressive curve can be obtained as illustrated in Fig. 9.

The leftest dotted line stands for the case where the diameter of the yarn does not vary. The righest dotted line

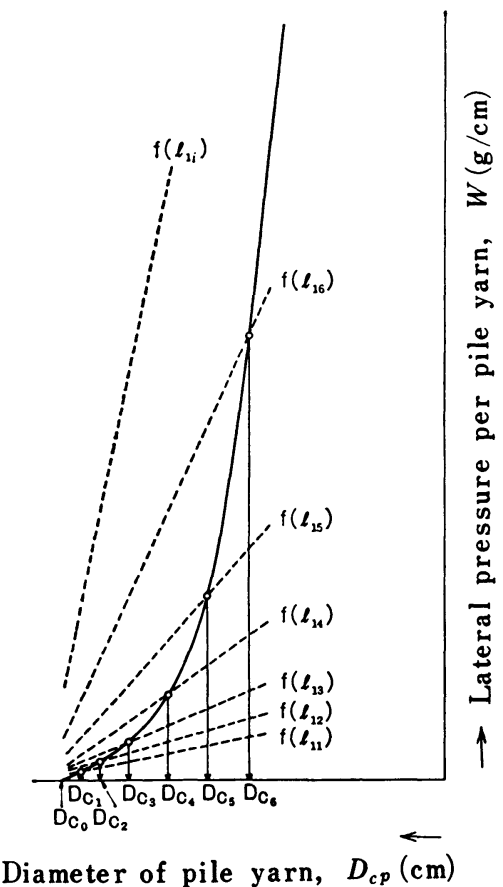

Fig. 8 Calculation of diameter of pile yarns under compression

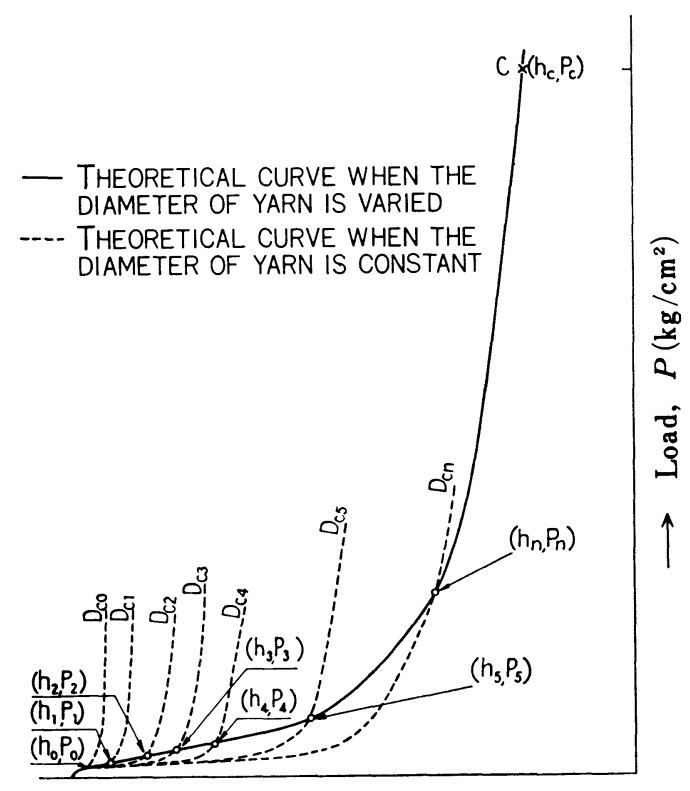

Thickness, $h(\mathrm{~cm}) \longleftarrow$

Fig. 9 Compressive deformation curves in which compresive effect of pile yarns is in corporated

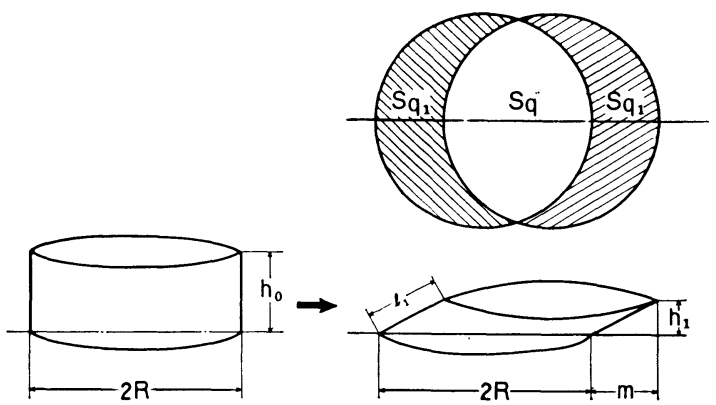

(a)

(b)

Fig.10 Adjusted model having small compressed area

is the case where the diameter of yarns is $D_{c n}$, i.e., completely collapsed from the beginning.

For the theoretical calculation of load-unload curve, the $\overrightarrow{E I}$ for compression and $\overleftarrow{E I}$ for unloading are used respectively.

2-2-3. When no neighboring piles exist

In general, this case does not occur. However, to prove the theory experimentally the disklike circular specimen having radius $R$ is compressed with a circular plate having radius $R c$ which is larger than $R$. Other part but the circular form was cut off.

If the carpet (a) in Fig. 10 is compressed and the piles are fallen down to (b), $m$ of eq. (24), i.e., $S q_{1}$, exerts no effect on the diameter of the pile yarns. In particular, it is necessary to adjust the effect of $S q_{1}$ when the compressed area 
is small considering the pile length. Putting the adjusted compressed area to $S q$, the following equation is geometrically obtained:

$$
S_{q}=(\pi R-2 m) R
$$

Hence, by putting $S q=S q o$, the lateral pressure $W(\mathrm{~g} / \mathrm{cm})$ per pile is as follows from eqs. (21) to (25),

$$
W=\frac{1}{c} \times \frac{P \sin ^{2} \alpha_{m}}{\sqrt{n}}
$$

where

$$
C=1-\frac{2 l_{1} \sin \alpha_{m}}{\pi R}
$$

As the diameter $R$ of the compressing plate increases, the adjusting coefficient $C$ reaches 1 . This is based upon the assumption that, while the portion $S q_{1}$ in Fig. 10(b) does not bear a compressive load, the rigidity of fibers or yarns may do. At the beginning of deformation, in which the bending rigidity of fibers acts effectively to bear the compressive force, the area $S q_{1}$ is comperatively small and the coefficient $C$ is nearly equal to 1 .

However, as the compressive deformation increases and the fibers are almost fallen down, the fallen-down yarns bear almost all the compressive load, and the bending rigidity in the region $S q_{1}$ can almost be neglected.

As both the area $S q_{1}$ and the $C$ increase, the adjustment becomes effective. This is the reason why the adjustment was made by neglecting the simple bending regidity.

2-3. Theoretical Calculation with completely fallen down piles

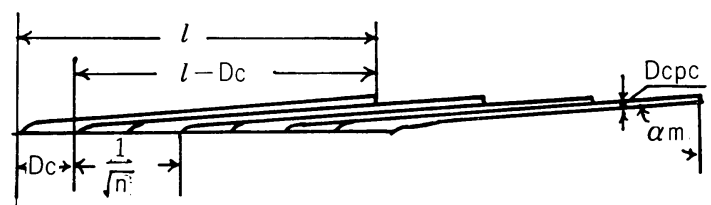

Fig. 11 Model of compressive deformation region at pile yarns

Fig. 11 shows the model after all the piles have fallen down. Assuming the compressive load in this case to be $P c\left(\mathrm{~g} / \mathrm{cm}^{2}\right)$, we have $\alpha_{m} \fallingdotseq \pi / 2$

Thus the lateral pressure $W c$ per pile is from eq. (26)

$$
W_{c}=\frac{P_{c}}{\sqrt{ } \bar{n}}
$$

From eq. (26) the adjusted lateral pressure is

$$
\begin{aligned}
& W_{c}=\frac{1}{C_{c}} \times \frac{P_{c}}{\sqrt{n}} \\
& \text { where, } C_{c}=1-\frac{2 l}{\pi R}
\end{aligned}
$$

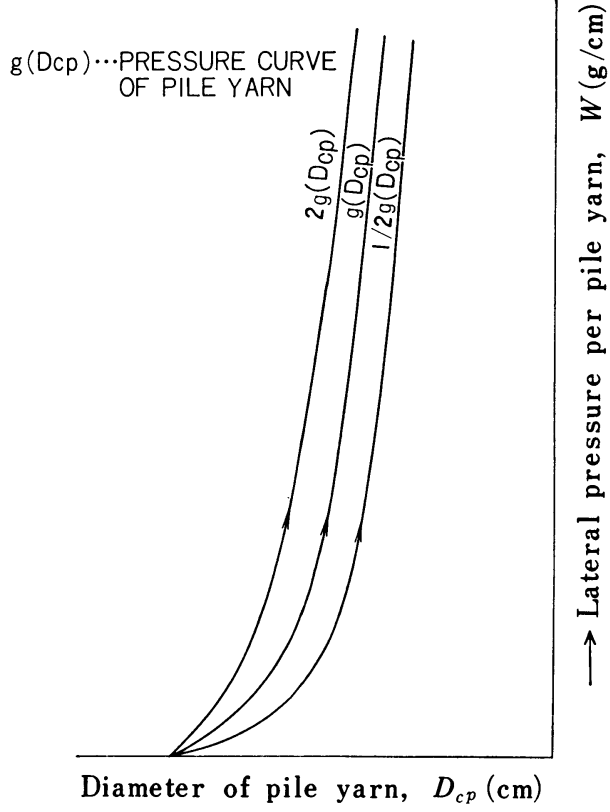

Fig. 12 Compressive property of pile yarn

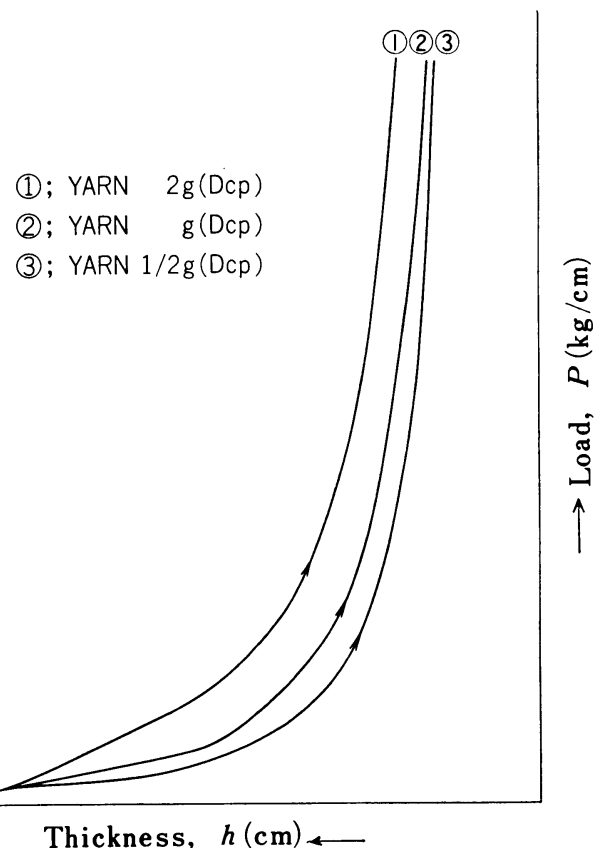

Fig. 13 The case where the compressive property of pile yarns varied

Now let us read the diameter of the yarn $D_{c p c}$ against $W c$ from the measured curve of the compressive property of the yarn.

The number of the overlapped yarns being $\left(l-D_{c}\right) \times \sqrt{n}$, the thickness $h c$ of the carpet at point $C$ is

$$
h_{c}=D_{c p c} \times\left(l-D_{c}\right) \times \sqrt{n}
$$


Thus $h c$ and $P c$ can be calculated, as illustrated in Fig. 9, and both the bending and the compressive deformation regions are connected.

2-4. Example of Calculation

2-3-1. When compressive properties of pile yarns vary

On the assumption that three kinds of carpets are made of pile yarns, same in structure but different in compressive properties, the above method is applied to the theoretical calculation, resulting in Fig. 13, which shows that the effect in the compressive deformation region of pile yarns is most influential.

\section{Experiments}

3-1. Device and Method

The same device and method that is reported in the previous article was used to plot the compressive deformation curves of piles of the carpet.

\section{3-2. Specimen}

Some of the most representative specimens are detailed in Table 1. The reason why these two carpets are used is that they are extremely different in compressive properties of the pile yarn when subjected to the lateral pressure, as illustrated in Fig. 14. Another reason is to see how this affects the compressive properties of the carpet, and also to make sure whether the theory can be applied to such different structure.

Table 1 Maria is of pile yarns

\begin{tabular}{c|c|c|c}
\hline & Count & Number of twists & Blend ratio \\
\hline Tufted carpet & $2 / 5.0$ & (s)96/(Z)272 & Wool $100 \%$ \\
\hline $\begin{array}{c}\text { Axminster } \\
\text { carpet }\end{array}$ & $2 / 4.85$ & (s)280/(Z)320 & Acryics $100 \%$ \\
\hline
\end{tabular}

From the construction view, the tufted carpets made of two-fold yarns illustrated in Figs. 15 and 16 have loose twists and no twist set. So, the tip of two-fold yarns when woven into the base fabric, the tip of two-fold yarns is liable to lose its twists and brings about the same result as the carpets made of single yarns. However, the aximinster carpet has bulky yarns with twist set. Table 2 shows the construction of the carpets.

Table 2 Constructions of carpets

\begin{tabular}{c|c|c|c|l}
\hline \hline & Pile length & $\begin{array}{c}\text { Pile inclination } \\
\text { angle }\end{array}$ & Stitch & Gauge \\
\hline Tufted carpet & $0.73 \mathrm{~cm}$ & $10^{\circ}$ & 8 piles/in & $1 / 8$ in \\
\hline $\begin{array}{c}\text { Axminster } \\
\text { carpet }\end{array}$ & 0.90 & 5 & 7 & $1 / 7$ \\
\hline
\end{tabular}

\section{3-3. Results of Measurement and Calculation}

Experiment was carried out at $20^{\circ} \mathrm{C} 65 \%$ R.H. with the compressed area of $\pi \mathrm{cm}^{2}$.

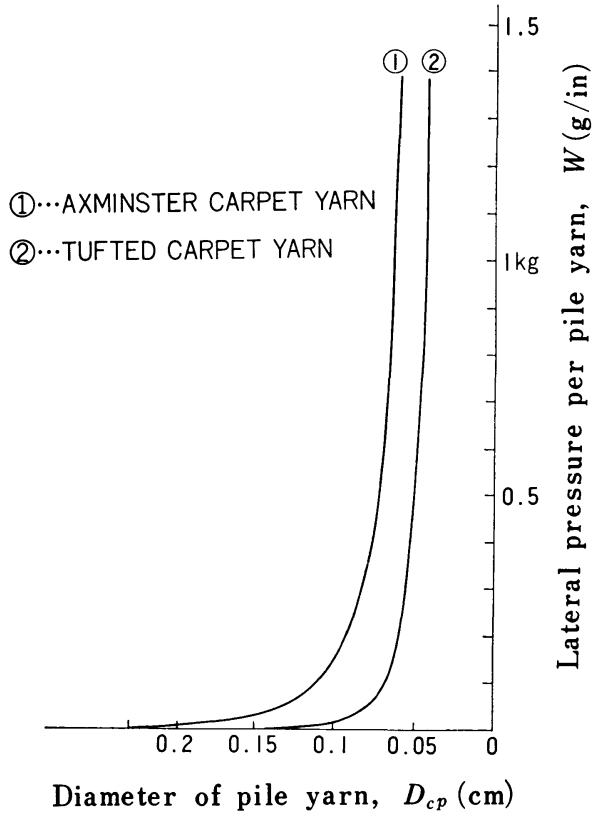

Fig. 14 Compressive property of pile yarn

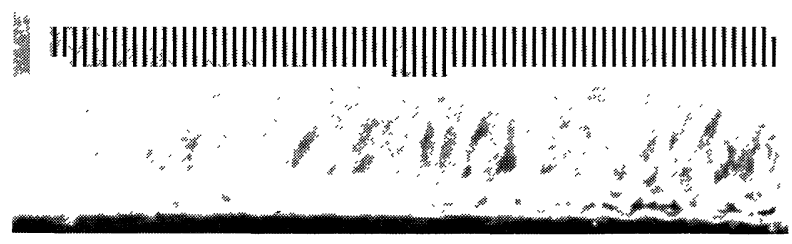

Fig. 15 Side view of tufted carpet

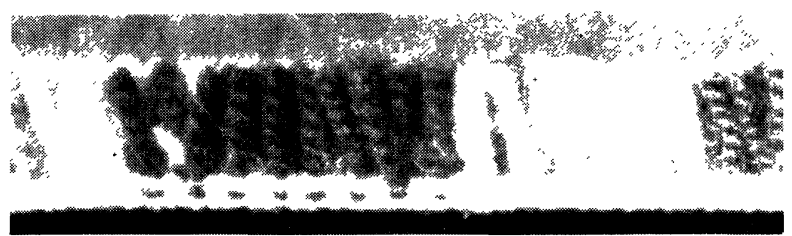

Fig. 16 Side view of Axminster carpet

Table 3 Example of Calculation

\begin{tabular}{|c|c|c|c|c|c|c|}
\hline \multirow[t]{2}{*}{$\begin{array}{ll}\cdots & D_{c p}\end{array}$} & \multicolumn{2}{|c|}{$0.125(\mathrm{~cm})$} & \multicolumn{2}{|c|}{$0.100(\mathrm{~cm})$} & \multicolumn{2}{|c|}{$0.080(\mathrm{~cm})$} \\
\hline & $\begin{array}{l}\pi P \\
(g)\end{array}$ & $W(g)$ & $\begin{array}{l}\pi P \\
(g)\end{array}$ & $W(\mathrm{~g})$ & $\begin{array}{l}\pi P \\
(g)\end{array}$ & $W(g)$ \\
\hline $0.1(\mathrm{~cm})$ & 421 & 29 & 444 & 33 & 458 & 34 \\
\hline 0.2 & 595 & 44 & 627 & 49 & 648 & 52 \\
\hline 0.3 & 904 & 71 & 953 & 81 & 984 & 85 \\
\hline 0.4 & 1535 & 131 & 1618 & 148 & 1671 & 157 \\
\hline 0.5 & 3161 & 293 & 3331 & 333 & 3441 & 354 \\
\hline 0.6 & 9896 & 1004 & - & - & - & - \\
\hline
\end{tabular}

The bending rigidity obtained per pile of the tufted carpet was $\overrightarrow{E I}=0.238 \mathrm{~g} . \mathrm{cm}^{2}$ and $\stackrel{\leftarrow E I}{E I}=0.059 \mathrm{~g} . \mathrm{cm}^{2}$, that of axminster carpets being $\overrightarrow{E I}=0.153 \mathrm{g.cm}{ }^{2}$ and $\stackrel{E}{E I}=$ 0.048 g.cm. 
Table 4 Example of caulation of compressive Deformation Region

\begin{tabular}{c|c|c|c|c|c|c|c}
\hline & $n$ & $l$ & $\pi P_{c}$ & $D_{c}$ & $W_{c}$ & $D_{c p c}$ & $h_{c}$ \\
\hline Tufted carpet & $\begin{array}{c}\text { piles } / \mathrm{cm}^{2} \\
19.8\end{array}$ & $0.73 \mathrm{~cm}$ & $3000 \mathrm{~g}$ & $0.135 \mathrm{~cm}$ & $401 \mathrm{~g}$ & $0.054 \mathrm{~cm}$ & $0.143 \mathrm{~cm}$ \\
\hline Axminster carpet & 15.1 & 0.90 & 3000 & 0.240 & 575 & 0.070 & 0.180 \\
\hline
\end{tabular}

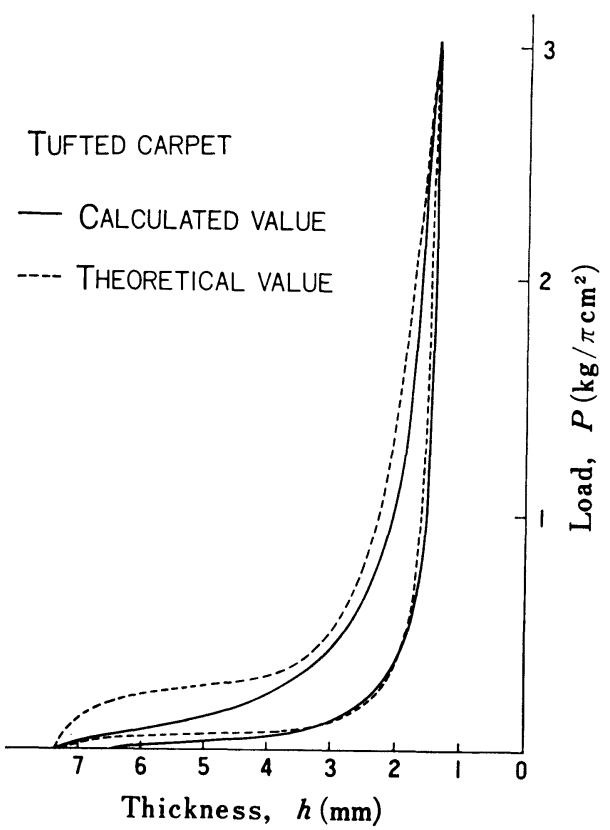

Fig. 17 Compressive deformation curves of tufted carpet

The compressive configuration was same as described in $2-1$, and $N_{A}=0.5$ and $N_{B}=0.5$. Also, from Table 2 and Fig. 14, the relation between $P$ and $h$ was calculated theoretically, and is shown in Tables 3 and 4.

They prove that the measured and theoretical values agree well, as illustrated in Figs. 17 and 18,

\section{Discussion}

The theory and the measurement above mentioned are based upon the ideal compressive configuration that the pile yarns fall down in the same direction when a load is imposed, and they showed good agreement.

When broad carpet is compressed with a small compressing plate as usually observed in the actual usage, the measured value differs from the theoretical value and shows high resilience. This should be further studied in detail.

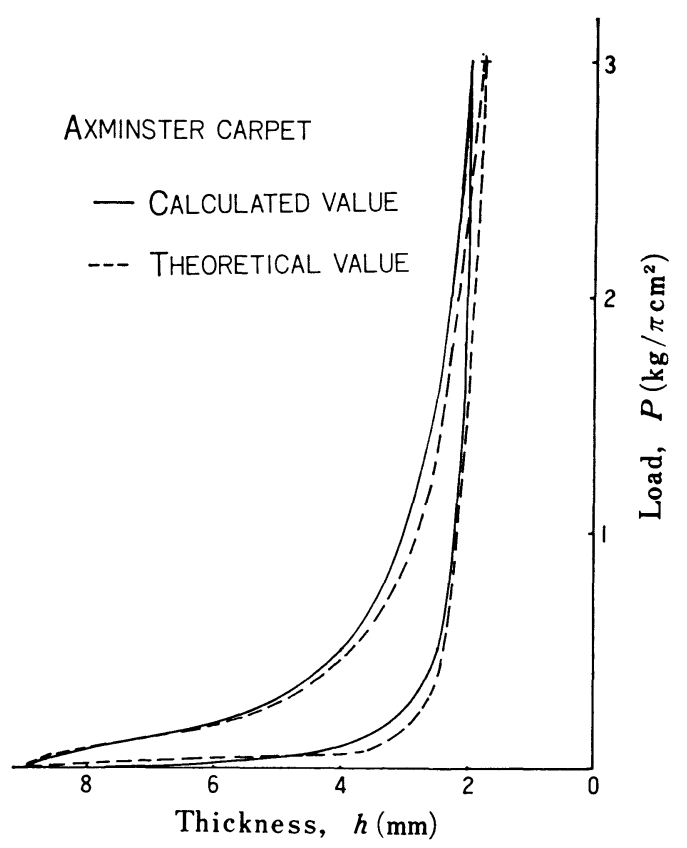

Fig. 18 Compressive deformation curves of Axminster carpet

\section{Conclusion}

By the improvement of the compressive deformation model of cut pile carpets, the compressive deformation curve of the cut pile carpets can be calculated from the mechanical properties of pile yarns, i.e., compressive properties and bending rigidity. The theory can be applied with accuracy to much broader range as compared with the old model reported in the previous article.

The theory has been already used for designing the compressive properties of carpets.

Thanks are due to the members of Fiber Assemblies Study Group in Dept. of Polymer Chemistry, Kyoto University, with whom the authors had many discussions.

\section{Literature cited}

[1] Kimura, Kawabata, Kawai; J. Text. Mach. Soc. Japan, 23, T67 (1970)

[2] Kimura, Kawabata; Pre-print presented at the 24th Annual Meeting of the Textile Machinery Society of Japan, p. 101 (1971) 